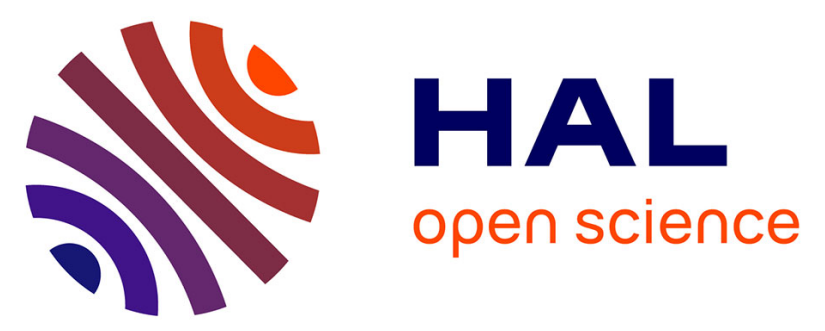

\title{
Minimizing the inter-vehicle distances of the time headway policy for urban platoon control with decoupled longitudinal and lateral control
}

Alan Ali, Gaëtan Garcia, Philippe Martinet

\section{- To cite this version:}

Alan Ali, Gaëtan Garcia, Philippe Martinet. Minimizing the inter-vehicle distances of the time headway policy for urban platoon control with decoupled longitudinal and lateral control. ITSC 2013 16th International IEEE Conference on Intelligent Transportation Systems, Oct 2013, The Hague, France. pp.1805-1810, 10.1109/ITSC.2013.6728490 . hal-02462061

\author{
HAL Id: hal-02462061 \\ https://hal.inria.fr/hal-02462061
}

Submitted on 31 Jan 2020

HAL is a multi-disciplinary open access archive for the deposit and dissemination of scientific research documents, whether they are published or not. The documents may come from teaching and research institutions in France or abroad, or from public or private research centers.
L'archive ouverte pluridisciplinaire HAL, est destinée au dépôt et à la diffusion de documents scientifiques de niveau recherche, publiés ou non, émanant des établissements d'enseignement et de recherche français ou étrangers, des laboratoires publics ou privés. 


\title{
Minimizing the inter-vehicle distances of the time headway policy for urban platoon control with decoupled longitudinal and lateral control
}

\author{
Alan ALI $^{1}$, Gaetan GARCIA ${ }^{2}$ and Philippe MARTINET ${ }^{1}$
}

\begin{abstract}
Recently in [1], a modification of classical constant time headway policy (CTH) was proposed in order to make CTH very practical and easy to use in real applications. This modification was tested and its benefits were shown only for highways application. In this paper, this modification is generalized in order to make it applicable in urban environment. Dynamic and kinematic models of the vehicle are mixted without accounting wheel slip. By using exact linearization technique, lateral and longitudinal dynamics become decoupled. Stability and accuracy of the global system are checked. Using TORCS [8] in simulation, effectiveness of the proposed modification and its potential effect on traffic density are reported.
\end{abstract}

\section{INTRODUCTION}

Nowadays, traffic congestion, related pollution, and safety problems become more and more important due to the explosion in the number of cars. Treatments of these problems on highways differ from those in urban areas. Compared with the urban areas, highways are characterized by high dynamics, faster cars, less curved roads, and less obstacles.

Driving automated vehicles in platoon is very effective in order to transport many passengers, to drive vehicles along main urban roads, to bring back vehicles to their base station, and it has many advantages such as increasing traffic density and safety, while decreasing fuel consumption and driver tiredness [11]. There is many projects in highways such as the platooning project in PATH program(Partners for Advanced Transit and Highways) [13], [14], SARTRE Project [12], and CHAUFFEUR project [6]. While the research are still goning on for urban applications such as V2I in LASMEA [2] .

Decoupling lateral and longitudinal dynamics is straightforward when using a static vehicle model [2]. On highway applications, with low curvature roads, and in urban applications with low speed, it is common to assume that the two dynamics are decoupled [14] [4]. Other researchers have built lateral and longitudinal controllers independently, the parameters of the lateral controller have been calculated for each speed [10].

The stability of platoon is defined as string stability, and it is given in [9], [14]. String stability means that the error must not amplify as it propagates through the platoon, and the errors must have the same sign to avoid collision.

The control can be global or local, according to intervehicle communications. Local control depends on data from adjacent vehicles, while global control depends on data,

1 A. ALI and P. MARTINET are with Institut de Recherche en Communications et Cyberntique de Nantes (IRCCYN), Ecole Centrale de Nantes $(\mathrm{ECN})$, Nantes, France

${ }^{2}$ G. GARCIA is with Ecole Centrale de Nantes (ECN), Nantes, France from at least the leader. In local control, the car is totally autonomous, it does not require sophisticated sensors, and can be used in all environments, but trajectory tracking and inter-vehicles distance keeping is not very accurate.

The desired inter-vehicle distances differ according to two control policies. In the first one we fix the distance between the vehicles (Constant spacing), and in the second one we change it according to vehicle's velocity or it's dynamic. Variable spacing can ensure string stability just by using onboard information [7], but the inter-vehicle distances is very large. Constant spacing can ensure string stability with high traffic density, but it requires inter-vehicle communications.

Constant time headway (CTH) is the simplest and most common among variable distance policies [14], [15], Others use variable time headway to reduce the distance between vehicles [17]. To author knowledge, all previous research has concentrated on optimizing the time headway for a good compromise between stability and inter-vehicle distance.

In this paper, we will deal with longitudinal and lateral control of platoons in urban areas. A dynamic and kinematic models will be proposed for longitudinal and lateral dynamics respectively. In the longitudinal dynamics, we take into account all the external forces applied on the car and the effect of lateral dynamics. In lateral dynamics, we will take steering dynamic into account, and we neglect wheel's slip as the vehicle travel on solid road (asphalt) and the maximum speed will be $50 \mathrm{~km} / \mathrm{h}$. This model might be first step to extend lateral model as have been done in [16]. Then, the model will be linearized (exact linearization) and the two dynamics will be totally decoupled. The longitudinal control law will depend on classical time headway policy with our modification. This modification makes the intervehicle distances proportional to velocity difference instead of being proportional to velocity. This new control law will be a mixture of local and global decentralized control. The string stability and control robustness will be verified. In the other hand, the lateral control will use sliding mode control to ensure the stability. Safety will be not discussed in this paper.

This paper is organized as follows: in section II models for the vehicle and platoon will be presented. Control will be developed in section III, string stability, and control robustness will be proved in section IV. Then in section V, we will show simulation results. Conclusion and perspective will be done in section VI. 


\section{Modeling}

\section{A. Kinematic Model Of The Vehicle}

We use classical car model (Ackerman model [3]). This model doesn't take slippage into account, and the car is represented as a bicycle as shown in figure (1). In general, this model can be used for light vehicles running at low speeds on sufficiently solid ground (asphalt).

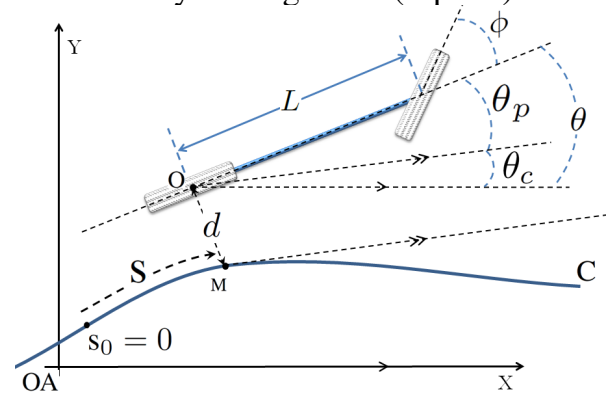

Fig. 1. Bicycle Model

where:

- $[O A, X, Y]$ is an absolute frame.

- $C$ is the common reference path.

- $O$ is the center of the rear wheel of the vehicle.

- $M$ is the closest point on $C$ to $O$.

- $s$ is the curvilinear coordinate of point $M$ along $C$, $c s=c(s)$ denotes the curvature of path $C$ at $M$, and $\theta_{c}(s)$ stands for the orientation of the tangent to $C$ at $M$, with respect to absolute frame.

- $\theta$ is the heading of the vehicle at point $O$, with respect to frame $[O A, X, Y]$.

- $\theta_{p}=\theta-\theta_{c}(s)$ denotes the angular deviation of the vehicle with respect to $C$.

- $d$ the lateral deviation of the vehicle with respect to $C$.

- $\phi$ is the steering angle, which is the angle between the front wheel and the body axis.

- $L$ is vehicle wheelbase.

- $v_{u}$ is the vehicle velocity along longitudinal vehicle axis.

- $N$ is the number of vehicles in the platoon.

The model is given by the following formula:

$$
\begin{aligned}
& \dot{s}=\frac{\cos \theta_{p}}{1-d \cdot c s} v_{u}, \dot{d}=\sin \theta_{p} \cdot v_{u} \\
& \dot{\theta}_{p}=\left(\frac{\tan \phi}{L}-\frac{c s \cdot \cos \theta_{p}}{1-d \cdot c s}\right) v_{u}
\end{aligned}
$$

This model has a single singularity when $d=\frac{1}{c s}$ (the vehicle is on the reference path curvature center). In practical situations, if the vehicles are well initialized, this singularity is never encountered.

The steering system is modeled as a first degree system [13]:

$$
\dot{\phi}=-\frac{1}{\tau_{s}} \phi+c_{2} \cdot u_{2}
$$

where $\phi$ is the front-steering angle , $\tau_{s}$ is the steering time constant, $c_{2}$ is a gain and $u_{2}$ is the vehicle's steering-anglecommand input.
By deriving $\dot{\theta}_{p}$ we get:

$$
\ddot{\theta}_{p i}=\alpha_{5 i} \dot{\phi}_{i}+\alpha_{6 i} \dot{v}_{u i}+\alpha_{7 i}
$$

where:

$$
\begin{aligned}
& \alpha_{5}=\frac{v_{u}}{L \cos ^{2} \theta_{p}}, \alpha_{6}=\frac{\tan \phi}{L}-\frac{c s \cos \theta_{p}}{1-d \cdot c s} \\
& \alpha_{7}=-\left(\frac{\dot{c} s \cdot \cos \theta_{p}}{1-d . c s}-\frac{c s \cdot \sin \theta_{p} \dot{\theta}_{p}}{1-d . c s}+\frac{c s \cdot \cos \theta_{p}}{(1-d . c s)^{2}}(\dot{d} . c s+d . \dot{c s})\right) v_{u}
\end{aligned}
$$

With the condition $\theta_{p} \neq \frac{\pi}{2}+\pi k, k=1,2 \ldots$

\section{B. Dynamic Model Of The Vehicle}

Dynamic equations for car-like robots with single or double steering can be found in [5]. We take the single steering dynamic model without slippage, figure (2). In addition, we will take into account drag, gravitational, and aero dynamic forces

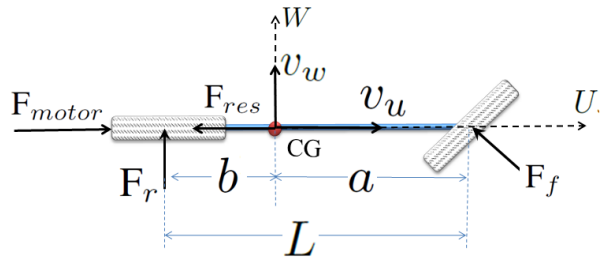

Fig. 2. Applied Forces

- $C G$ : vehicle's center of gravity.

- $[C G, U, W]$ : vehicle frame.

- $v_{w}$ is vehicle velocity at point $C G$ along w axis.

- $F_{g}, F_{a e r o}, F_{d r a g}$ : Gravitational, aero dynamical and mechanical forces respectively.

- $g$ : Acceleration of gravity.

- $\xi$ : Angle between the road surface and horizon.

- $\rho$ : Specific mass of air, $A$ : Cross-sectional area of the vehicle. $C_{d}$ : Vehicle's drag coefficient.

- $d_{m}$ : Mechanical drag.

- $F_{f}, F_{r}$ : are the resultant lateral forces on the front and rear tires respectively.

- $F_{\text {motor }}$ : is the driving force applied at the rear axle (first system input $u_{1}$ ).

- $F_{\text {res }}=F_{\text {drag }}+F_{\text {aero }}+F_{g}$

- $m$ : mass of the vehicle

- $J$ : mass moment of inertia of the vehicle about the center of gravity.

The following dynamic equations is taken from [5] with some changes to take into account the effects of aero dynamic, drag and gravity forces represented by $F_{\text {res }}$ :

$$
\begin{aligned}
& \dot{v}_{u}=v_{w} \dot{\theta}-\frac{F_{f} \sin \phi}{m}+\frac{F_{\text {motor }}+F_{\text {res }}}{m} \\
& \dot{v}_{w}=-v_{u} \dot{\theta}-\frac{F_{f} \cos \phi}{m}+\frac{F_{r}}{m} \\
& \ddot{\theta}=\frac{a F_{f} \cos \phi}{J}-\frac{b F_{r}}{J}
\end{aligned}
$$

and $F_{r e s}$ is given by:

$$
F_{\text {res }}=-m g \sin \xi-\frac{\rho A C_{d}}{2} v_{u}^{2} \operatorname{sgn}\left(v_{u}\right)-d_{m}
$$


The nonholonomic constraints are:

$$
v_{w}=\dot{\theta} b \quad, \quad \dot{\theta}=\frac{\tan \phi}{L} v_{u}
$$

The derivatives of the nonholonomic constraints are:

$$
\dot{v}_{w}=\ddot{\theta} b \quad, \quad \ddot{\theta}=\frac{\tan \phi}{L} \dot{v}_{u}+\frac{v_{u}}{L \cdot \cos ^{2} \phi} \dot{\phi}
$$

Developing the dynamical equations (4) with nonholonmoic equations(6) and their derivatives (7), this gives the longitudinal dynamic equation taking into account lateral dynamic effects:

$$
\dot{v_{u}}=\alpha_{1}\left(u_{1}+F_{r e s}\right)+\alpha_{2} \dot{\phi}
$$

where :

$$
\begin{aligned}
& \alpha_{1}=\frac{L^{2}}{\left(L^{2} m+\left(b^{2} m+J\right) \tan ^{2} \phi\right)} \\
& \alpha_{2}=\frac{\left(b^{2} m+J\right) \tan \phi}{\cos ^{2} \phi \cdot\left(L^{2} m+\left(b^{2} m+J\right) \tan ^{2} \phi\right)} v_{u}
\end{aligned}
$$

with the condition $\phi_{i} \neq \frac{\pi}{2}+\pi k, k=1,2 \ldots$ This condition is always satisfied because the maximum steering angle is limited and it is always smaller than $\frac{\pi}{2}$.

\section{Platoon Model}

The platoon consists of many vehicles following each other. The first vehicle is the leader, it may be driven manually or automatically. The other vehicles follow each other, moving at the same speed and keeping a desired distance between each two consecutive vehicles as shown in figure(3). We define the following curvilinear parameters assuming a point mass model for all vehicles:

- $l_{i}$ : Desired constant curvilinear inter-vehicle distance.

- $s_{i}$ : Curvilinear coordinate of $i^{\text {th }}$ vehicle.

- $\dot{s}_{i}$ : Curvilinear Velocity of the $i^{t h}$ vehicle.

- $e s_{i}=s_{i-1}-s_{i}-l_{i}$ : Curved spacing error of the $i^{t h}$ vehicle.

As we will see in the control section, we will decouple vehicles dynamics into two decoupled dynamics. The control law for each dynamics will be represented using springdamper model.

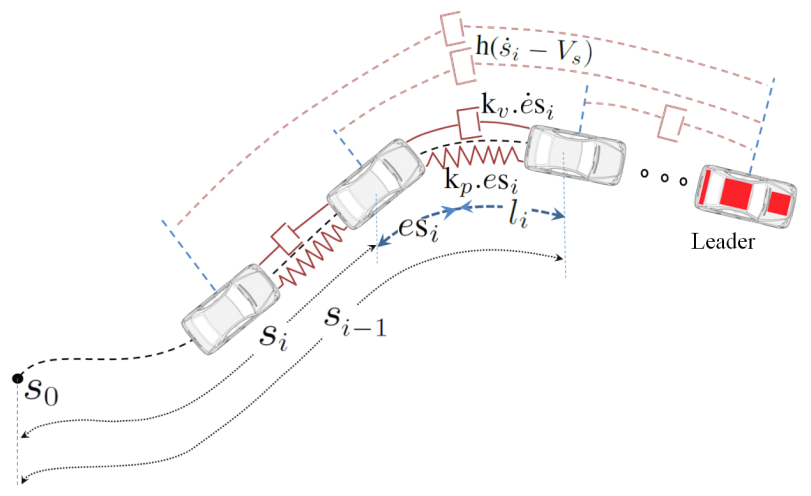

Fig. 3. Longitudinal Platoon Model

The longitudinal model shown in figure(3) consists of one spring and two dampers. The amplitudes of the spring depends on the curvilinear inter-vehicle distances, this act as attraction force when the inter-vehicle distance is bigger than desired distance, and as repulsive force when it is smaller. The Amplitudes of first damper (shown in solid line) depends on curvilinear velocity difference between each two consecutive vehicles, this damper act as movement damper. The second damper (shown in dash line) depends on the difference between curvilinear vehicle and a reference velocity (leader velocity in this figure), this damper will assure platoon stability, it is shown in dash style to indicate that the reference velocity is updated each many samples time.

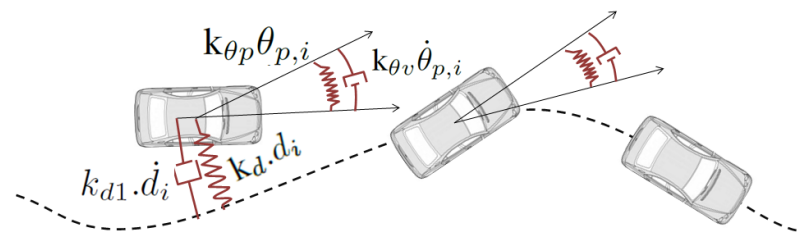

Fig. 4. Lateral Platoon Model

In the lateral model there are two springs and two dampers. The amplitude of the angle spring depends on the angle error $\theta_{p i}$, it works as attractive moment toward zero. The damper amplitude depends on the speed of changing of this angle $\left(\dot{\theta}_{p i}\right)$. Finally the amplitude of the second spring is proportional to the lateral distance error $d$, and the second damper to its changes $\dot{d}$.

\section{CONTROL}

\section{A. Control Objectives}

The main objectives of the control law are to:

1) Keep the curvilinear inter-vehicle distance equal to $l$, and to make all vehicles move at the same speed so $\dot{e} s_{i}=0$ (longitudinal control).

2) Follow the desired path by making $\theta_{p}=0$ and $d=0$ (lateral control).

3) Assure string stability of the platoon (spacing error must decrease as it propagates through the platoon).

4) Increase traffic density, using minimum required data from other vehicles in the platoon.

5) Keep system stability even in case of total communication loss.

6) Accommodate the instability caused by the actuation and sensing lags (robustness).

\section{B. Decoupling And Exact Linearization}

The total model of the $i^{\text {th }}$ vehicle is:

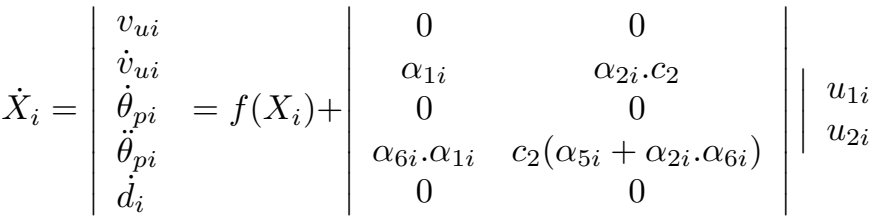

$$
\begin{aligned}
& Y_{i}=\left|\begin{array}{l}
y_{1 i} \\
y_{2 i}
\end{array}=\right| \begin{array}{l}
s_{i} \\
\theta_{p i}
\end{array}
\end{aligned}
$$


We can linearize the system by static feedback linearization by taking new inputs $w_{1 i}, w_{2 i}$ as the follow:

$$
y_{1 i}^{(r 1)}=\dot{v}_{u i}=w_{1 i}, y_{2 i}^{(r 2)}=\ddot{\theta}_{p i}=w_{2 i}
$$

with the condition $v_{u} \neq 0$. So, this control law is not good for very low speed velocities.

We choose new coordinates and we get the following linear system:

$$
\left|\begin{array}{c}
v_{u i} \\
\dot{v}_{u i} \\
\dot{\theta}_{p i} \\
\ddot{\theta}_{p i}
\end{array}=\right| \begin{aligned}
& \dot{Z}_{1} \\
& \dot{Z}_{2} \\
& \dot{Z}_{3} \\
& \dot{Z}_{4}
\end{aligned}=\left|\begin{array}{llll}
0 & 1 & 0 & 0 \\
0 & 0 & 0 & 0 \\
0 & 0 & 0 & 1 \\
0 & 0 & 0 & 0
\end{array}\right|\left|\begin{array}{l}
Z_{1} \\
Z_{2} \\
Z_{3} \\
Z_{4}
\end{array}+\right| \begin{array}{ll}
0 & 0 \\
1 & 0 \\
0 & 0 \\
0 & 1
\end{array}|| w_{1 i}
$$

The remainder non linearized part represents the internal dynamic of the system:

$$
\dot{Z}_{5}=\dot{d}_{i}=\sin \left(Z_{3}\right) \cdot Z_{1}
$$

The zero dynamic of this system is stable.

\section{Longitudinal Control}

We take the classical time headway policy and we add a new modification that will enhance system's response. The new modification is shown in red dash line in figure (5).

We propose the following new curvilinear spacing error:

$$
\delta_{i}=e s_{i}-h\left(\dot{s}_{i}-V_{s}\right)
$$

where $V_{s}$ is a common velocity value shared by all vehicles of the platoon, it must be the same value for all the vehicles at the same sampling time.

The control law is:

$$
w_{1 i}=\frac{\dot{e s} s_{i}+\lambda \delta_{i}}{h}
$$

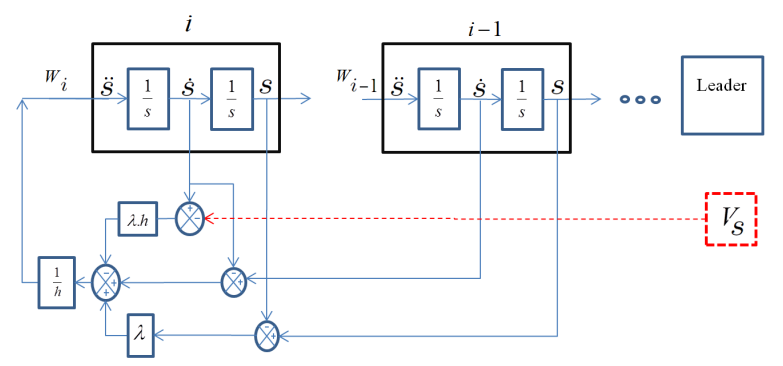

Fig. 5. Longitudinal Control diagram

\section{Lateral Control}

We define sliding surface as follows:

$$
\psi=\dot{\theta}_{p i}+k_{\theta p} \theta_{p i}+k_{d} d i
$$

where $k_{\theta p}, k_{d}$ are weighting coefficients. The controller should provide an input that satisfies the following:

$$
\dot{\psi}=-K \psi
$$

with $K$ positive constant.

So from equations (13)(12) and (9), we get the following control law:

$$
w_{2 i}=-K \psi-k_{\theta p} \dot{\theta}_{p i}-k_{d} \dot{d}_{i}
$$

which gives a stable system.

\section{STABILITY AND ROBUSTNESS}

\section{A. String Stability of Longitudinal Control}

String Stability for a platoon means that the spacing error must decrease as it propagates through the platoon [9]:

$$
\left\|e s_{i}\right\|_{\infty} \leq\left\|e s_{i-1}\right\|_{\infty}
$$

To verify this condition, we define the spacing error propagation transfer function $G_{i}(p)=\frac{e s_{i}(p)}{e s_{i-1}(p)}$. A sufficient condition for string stability is:

$$
\left\|G_{i}(p)\right\|_{\infty} \leq 1 \quad \text { and } \quad g_{i}(t)>0 \quad i=1,2 . . N
$$

where $g_{i}(t)$ is error propagation impulse response of the $i^{\text {th }}$ vehicle.

In our case, the propagation transfer function $G_{i}(p)$ of the curvilinear error is:

$$
G_{i}(p)=\frac{e s_{i}(p)}{e s_{i-1}(p)}=\frac{1}{h \cdot p+1}
$$

After some developments, it can be proved that:

$$
\left\{\left|G_{i}(\omega)\right|=\frac{1}{\sqrt{h^{2} \omega^{2}+1}} \leq 1, \quad g_{i}(t)=\frac{e^{\frac{-t}{h}}}{h}>0\right.
$$

So the system is string stable, because the curvilinear spacing errors, at all frequencies, do not increase as they propagate through the platoon. We can see that the string stability is independent from $V_{s}$, and the only conditions is to share the value for all the vehicles at the same sampling time. So we can choose any value for V (e.g. leaders velocity, the medium velocity of the platoon or the minimum velocity in the platoon...).

\section{B. Robustness to Unmodeled Actuation and Sensing Lags}

Approximations made while modeling dynamic systems may hide some system dynamics This unmodeled dynamics may appear as lags in actuator response. In the other hand, filtering sensors inputs is the main source of sensing lags. So, it is required to study the effect of this lags on the control to verify its robustness. Actuators and sensing lags can be modeled as follows [15]:

$$
\begin{gathered}
\ddot{x}_{i}=w_{c i} \\
\tau \dot{w}_{c i}+w_{c i}=w_{i}
\end{gathered}
$$

where: $\tau$ represents lag time constant. $w_{c i}$ is the acceleration of the $i^{t h}$ vehicle, and $w_{i}$ is control input. We have modeled all the lags in the system in the second equation in an aggregate manner.

The new spacing error propagation transfer function $G_{p}$ can be computed from equation (18) and the same equation for the $(i-1)^{t h}$, we get:

$$
G_{p}(p)=\frac{e s_{i}}{e s_{i-1}}=\frac{p+\lambda}{\tau h p^{3}+h p^{2}+(1+\lambda h) p+\lambda}
$$

To assure the string stability for this disturbed system, we have to ensure $\left|G_{p}(j . \omega)\right| \leq 1$ for all $\omega$. So we get the following:

$$
\tau^{2} h^{2} \omega^{4}+\left(h^{2}-2 \tau h(1+\lambda h)\right) \omega^{2}+\lambda^{2} h^{2} \geq 0
$$


The above inequality holds only if the discriminant is negative or if the coefficient of $\omega^{2}$ is positive. Both conditions are satisfied [14] only if

$$
\tau \leq \frac{h}{2}
$$

This means, the sum of all unmodeled lags must be less than or equal to half the time headway employed.

\section{SIMULATIONS}

Simulation has been done using Matlab and TORCS. TORCS (The Open Racing Car Simulator [8]) is one of the most popular car racing simulators for academic purposes. It features a sophisticated physics engine (aerodynamics, fuel consumption, traction...) as well as a 3D graphics engine for the visualization of the races shown in figure (6).
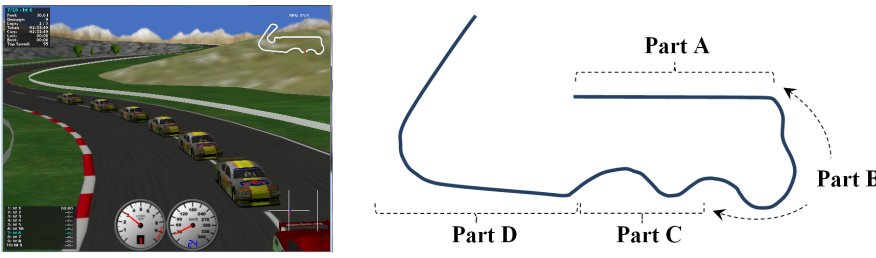

Fig. 6. TORCS window and test track

Simulation will be done on Torcs using curved track shown in figure(6), to test the stability of lateral and longitudinal control laws. A platoon of 10 identical cars will moves on curved track. The first part of this track is nearly straight (part A), so we will test the longitudinal string stability by changing the velocity from $10 \mathrm{~km} / \mathrm{h}$ to $50 \mathrm{~km} / \mathrm{h}$. Then the platoon will pass the curved parts (part B) with fixed speed to verify lateral stability. Finally we will try to verify the stability of both control together by changing the speed while passing curved part, so we change the velocity from $50 \mathrm{~km} / \mathrm{h}$ to $25 \mathrm{~km} / \mathrm{h}$ while passing part $\mathrm{C}$, and we increase the velocity to $60 \mathrm{~km} / \mathrm{h}$ while passing the road corner at the beginning of part $\mathrm{D}$.
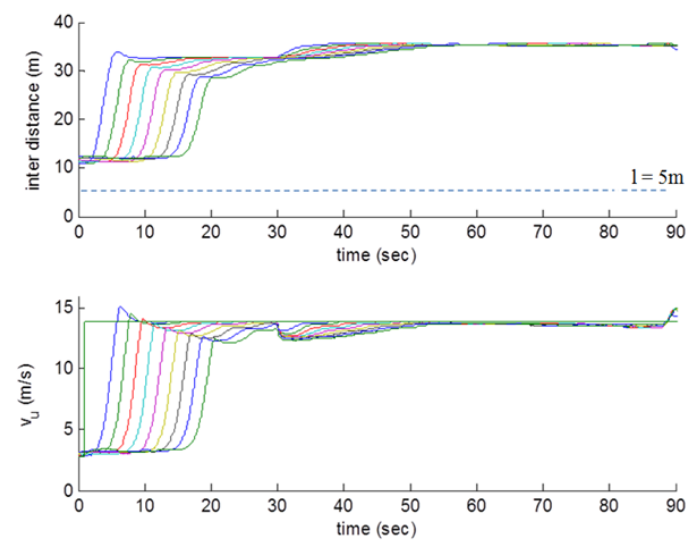

Fig. 7. inter-vehicle distances and vehicle's velocities for classical time headway policy.

Firstly, we compare our longitudinal control law with the classical constant time headway law for urban areas.
Figure(7) show the curved inter-vehicle distances using the classical time headway law, and figure(8) show this distances using our control law using the same parameters. We can see that the inter-vehicle distances using our law have been minimized to become equal to desired distance in steady state, and proportional just to velocity difference in transient phase. In addition, in the worst case when the first error is at its maximum value (which is the biggest maximum) the others errors aren't at the maximum values, so the platoon length expansion will be smaller than the sum of maximum errors of all the vehicles. We can see also that our control law makes the error stabilization faster. So, all other results obtained in [1] can be generalized to urban areas, so our law keeps the same stability of classical law, at the same time it minimizes the required inter-vehicle distances. It dosen't require so much information from other vehicles because we can update $V_{s}$ value each many sample time, and the system can be also stable even if the communication is totally lost. What's more, our control law is robust to actuator and sensors lags, and we can easily avoid torque saturation by increasing $h$ with small expansion in platoon length in transient phase. Finally, all results are obtained using very simple control law (modified time headway policy) and require simple data (velocity) at low frequency.
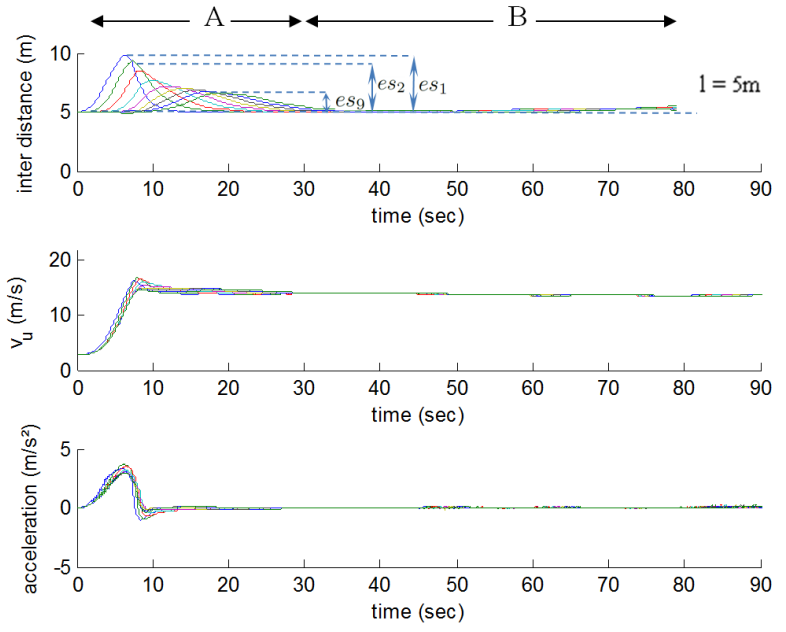

Fig. 8. inter-vehicle distances and vehicle's velocities(part A,B).

Secondly, we verify the stability and the effectiveness of decoupling the two controls laws. We can see in figure(8) in part A (the straight part) that the system is string stable, because the inter-vehicle distance error decreases as it propagates through the platoon, and the final inter-vehicle distance is equal to desired distance $l$.

In figure(9) in part B, we can see that the lateral control is stable and lateral errors(angle and distance) and decreases to zero. Values are very small (angle error $<3 \mathrm{deg}$ and distance error $<20 \mathrm{~cm}$ ), and we can see that in this part that the lateral control has very small effect on the inter-vehicle distances, which still equal to desired distance. Finally in part D, in figures (10 and 11), we test the two control together. We can see that the system is string stable in longitudinal control, and the lateral control also is stable. 

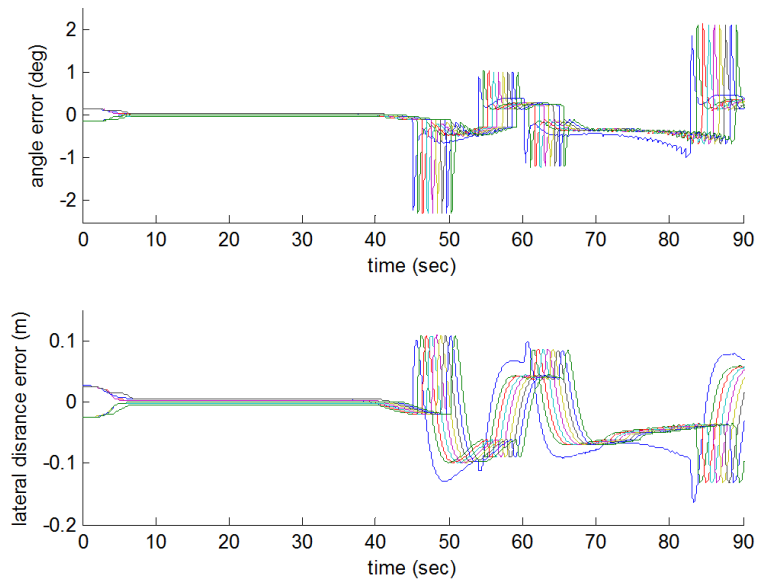

Fig. 9. Lateral angle and distance errors(part A,B).
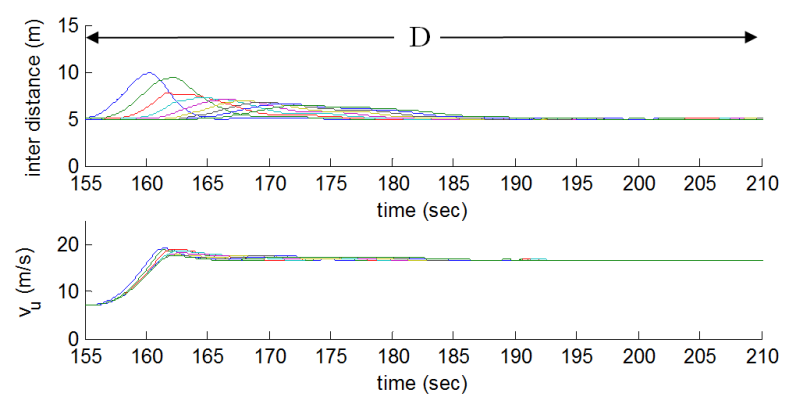

Fig. 10. inter-vehicle distances and vehicle's velocities(part D).

\section{CONCLUSION}

In this paper we have addressed the control of platoons in urban areas. A dynamical longitudinal model and kinematic lateral model are used and have been decoupled and linearized. The decoupling effectiveness has been verified. The lateral dynamic is controlled using sliding mode control and we have got very accurate performance. The longitudinal dynamic is controlled using modified CTH control law. We have generalized this modified control law to be applicable in urban application because it has many advantages. These advantages were detailed in [1] and can be summarized by the following. It minimizes the inter -vehicle distance, which were very big and considered the main shortcoming of classical CTH policy. It also enables to increase the stability, the robustness, and avoid torque saturation. All these benefits are obtained without the need for high rate transfer data from other vehicles. Moreover, the platoon can still be stable even if the communication link is totally lost. This modification open the door to move CTH policy from research to real applications, after we have made the inter-vehicle distance become nearly equal to constant spacing policy.

\section{REFERENCES}

[1] Ali A., Garcia G., and Martinet P., Minimizing the inter-vehicle distances of the time headway policy for platoons control in highways, to be published in 10th International Conference on Informatics in
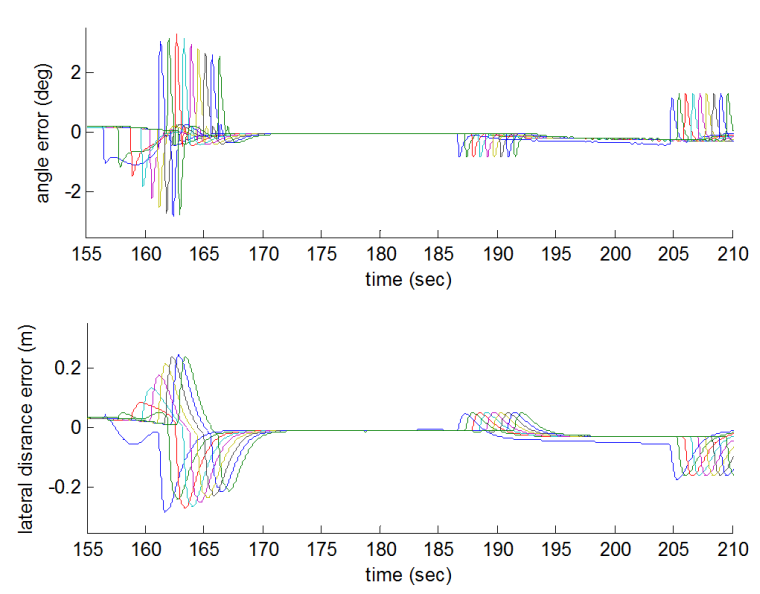

Fig. 11. Lateral angle and distance errors(part D).

Control, Automation and Robotics (ICINCO13), Reykjavik, Iceland, July 29-31, 2013.

[2] Bom J., Thuilot B., Marmoiton F., and Martinet P., Nonlinear control for urban vehicles platooning, relying upon a unique kinematic gps. In Proceedings of IEEE International Conferenceon Robotics and Automation, pp. 4138- 4143, Barcelonna, Spain, 2005.

[3] Canudas de Wit C., Siciliano B., Bastin G., Theory of Robot Control, Series: Communications and Control Engineering, Springer, ISBN 3540760547, 1996

[4] Daviet P., Parent M., Longitudinal and lateral servoing of vehicles in a platoon, Proceedings of IEEE Intelligent Vehicles Symposium, pp.41-46, 19-20 September 1996.

[5] DeSantis R.M., Path-tracking for car-like robots with single and double steering, IEEE Transactions on Vehicular Technology, vol.44, no.2, pp.366,377, May, 1995.

[6] Fritz H., Gern A., Schiemenz H., Bonnet C., CHAUFFEUR Assistant: a driver assistance system for commercial vehicles based on fusion of advanced ACC and lane keeping, 2004 IEEE on Intelligent Vehicles Symposium, pp.495,500, 14-17 June, 2004

[7] Ioannou P., Chien C., Autonomous intelligent cruise control, IEEE Transactions on Vehicular Technology, 42(4):657 -672, 1993.

[8] Onieva E., Pelta D., Alonso J., Milanes V., Perez J., A modular parametric architecture for the torcs racing engine. In IEEE Symposium on Computational Intelligence and Games, pp. 256 -262, 2009.

[9] Rajamani R., Vehicle dynamics and control, Springer science, ISBN 0387263969, 2006.

[10] Rajamani R., Han-Shue Tan, Boon Kait Law, Wei-Bin Zhang, Demonstration of integrated longitudinal and lateral control for the operation of automated vehicles in platoons, IEEE Transactions on Control Systems Technology, vol.8, no.4, pp. 695,708, Jul 2000.

[11] Ricardo. http://www.ricardo.com/en-GB/News-Media/Pressreleases/News-releases1/2009/Cars-that-drive-themselves-canbecome-reality-within-ten-years/, 2009.

[12] SARTRE-Consortium (2009 to 2012). Sartre project. http://www.sartre-project.eu/en/Sidor/default.aspx.

[13] Sheikholeslam S., Desoer C.A., Combined Longitudinal and Lateral Control of a Platoon of Vehicles, American Control Conference, 1992 , pp. 1763,1767, 24-26 June. 1992.

[14] Swaroop D., String stability of interconnected systems: An application to platooning in automated highway systems. UC Berkeley: California Partners for Advanced Transit and Highways (PATH), 1997.

[15] Swaroop D., Rajagopal K., A review of constant time headway policy for automatic vehicle following. In Proceedings IEEE Intelligent Transportation Systems, pp. $65-69,2001$.

[16] Lenain R., Thuilot B., Hach O., Martinet P., High-speed mobile robot control in off-road conditions: A multi-model based adaptive approach, IEEE International Conference on Robotics and Automation (ICRA), pp. 6143,6149. 9-13, May. 2011.

[17] Yanakiev D., Kanellakopoulos I., Variable Time Headway for String Stability of Automated Heavy-Duty Vehicles, Proceedings of the 34th IEEE Conference on Decision and Control, 1995, pp. 4077-4081, 1995. 